\title{
Lymphedema Associated With Primary Amyloidosis: A Case Study
}

\author{
Gahee Park, $\mathrm{MD}^{1}$, Hye Won Jeong, $\mathrm{MD}^{1}$, Junhee Lee, $\mathrm{MD}^{1}$, Yeung-Chul Mun, $\mathrm{MD}, \mathrm{PhD}^{2}$, \\ Sun Hee Sung, $\mathrm{MD}, \mathrm{PhD}^{3}$, Soo Jeong Han, $\mathrm{MD}, \mathrm{PhD}^{1}$
}

Departments of ${ }^{1}$ Rehabilitation Medicine, ${ }^{2}$ Internal Medicine, and ${ }^{3}$ Pathology, Ewha Womans University School of Medicine, Seoul, Korea

\begin{abstract}
We reported on a 60 -year-old man presenting lymphedema of both lower extremities and scrotum for 3 years with unknown cause. We took a computed tomography scan of the lower extremities as a follow-up. There were diffuse subcutaneous edema in both lower extremities and multiple enlarged lymph nodes along the para-aortic and bilateral inguinal areas. For further evaluation, biopsy of an enlarged inguinal lymph node was taken, yielding a diagnosis of primary amyloidosis. A treatment of chemotherapy for amyloidosis was recommended for him. To our knowledge, this is the first report of lymphedema presenting with primary amyloidosis in Asia. This case suggests that primary amyloidosis could be one of the differential diagnoses in patients with lymphedema in the lower extremities.
\end{abstract}

Keywords Lymphedema, Amyloidosis, Lymph nodes

\section{INTRODUCTION}

Amyloidosis is a rare disease caused by deposition of protein fibrils in extracellular areas. Of the types of amyloidosis, amyloid light chain amyloidosis (AL, primary amyloidosis) is more common than amyloid A protein amyloidosis (AA, secondary amyloidosis) [1]. The incidence of AL amyloidosis was reported as 3 to 5 cases per $1,000,000$ population [2]. In amyloidosis patients, the most commonly affected organ is the kidney. AL Amyloi- dosis patients usually had proteinuria in the nephrotic range along with hypoalbuminemia. The second most commonly involved organ is the heart, where amyloidosis manifests as restrictive cardiomyopathy, such as with thickened ventricles or diastolic dysfunction. AL amyloidosis can also affect other organs, including the respiratory tract, bladder, and soft tissue. Advanced and irreversible organ dysfunction has often occurred by the time a clinical diagnosis of amyloidosis is made, so a highly suspicious inspection is important for early diagnosis [1].

Received September 21, 2016; Accepted November 8, 2016

Corresponding author: Soo Jeong Han

Department of Rehabilitation Medicine, Ewha Womans University School of Medicine, 1071 Anyangcheon-ro, Yangcheon-gu, Seoul 07985, Korea. Tel: +82-2-2650-5035, Fax: +82-2-2650-5145, E-mail: ocrystal@ewha.ac.kr

ORCID: Gahee Park (http://orcid.org/0000-0002-5133-2449); Hye Won Jeong (http://orcid.org/0000-0001-9186-0943); Junhee Lee (http://orcid. org/0000-0001-7722-9979); Yeung-Chul Mun (http://orcid.org/0000-0002-1882-3983); Sun Hee Sung (http://orcid.org/0000-0001-9345-1131); Soo Jeong Han (http://orcid.org/0000-0002-5685-0384).

(c) This is an open-access article distributed under the terms of the Creative Commons Attribution Non-Commercial License (http://creativecommons.org/ licenses/by-nc/4.0) which permits unrestricted noncommercial use, distribution, and reproduction in any medium, provided the original work is properly cited. Copyright $\odot 2017$ by Korean Academy of Rehabilitation Medicine 
Lymphedema is the swelling of tissue caused by inadequate lymphatic circulation. Secondary lymphedema is more common than primary lymphedema and is caused by an obstruction of the lymphatic system, which can be due to malignancy, infection, trauma, or surgery [3]. Lymphedema decreases quality of life, and has an irreversible progression.

We report on a patient with lymphedema in both lower extremities as a complication of primary amyloidosis with the involvement of multiple lymph nodes along the para-aortic and bilateral inguinal areas.

\section{CASE REPORT}

A 60-year-old man complained of swelling, discharge, pain, and tenderness on both legs. He had a medical history of hypertension, diabetes mellitus, and obesity (body mass index, $49.5 \mathrm{~kg} / \mathrm{m}^{2}$ ), and reported in his life history as having lived in Taiwan for one year, 12 years ago. Localized non-pitting edema in both lower extremities occurred 3 years ago. He had visited our clinic several times for complex decongestive therapy and medication for intermittent pain.

In recent visits, cellulitis in both lower extremities and lymphedema on the scrotum were observed, with clinical presentation of urinary frequency and nocturia. There were yellowish discharge, foul odor, nodular skin changes, and elephantiasis in both lower extremities. A filariasis antibody test was administered to rule out lymphedema filariasis because of his residence history; but the result was negative. Laboratory tests showed normal serum creatinine level, glomerular filtration rate, and $\mathrm{N}$-terminal pro-B-type natriuretic peptide (NT-proBNP). M-protein was not found in serum and urine electrophoresis (Table 1). To exclude cardiopulmonary dysfunction, electrocardiograms, a transthoracic echocardiography, and a pulmonary embolism computed tomography (CT) scan were performed; no specific finding was shown.

Follow-up abdomen CT, pelvis CT, and lower extremities CT scans showed no evidence of thrombosis; however, there were multiple enlarged lymph nodes along the para-aortic and bilateral inguinal areas (Fig. 1), which were not shown on the previous lower extremities CT scan taken 3 years ago. Also, lymph nodes in aortocaval, para-aortic, common iliac, external iliac, inguinal, and femoral areas were enlarged in abdomen and pelvis CT.
Table 1. Results of the patient's blood test

\begin{tabular}{lc}
\hline \multicolumn{1}{c}{ Parameter } & Value \\
\hline $\mathrm{Hb}(\mathrm{g} / \mathrm{dL})$ & 10.1 \\
\hline $\mathrm{WBC}(/ \mathrm{L})$ & $6.90 \times 10^{9}$ \\
Platelet (/L) & $304 \times 10^{9}$ \\
Sodium (mEq/L) & 134 \\
Potassium (mEq/L) & 5.3 \\
Calcium (mg/dL) & 7.4 \\
\hline BUN (mg/dL)/Creatinine (mg/dL) & $12 / 0.95$ \\
Total protein (g/dL) & 7.2 \\
CRP (mg/dL) & 10.58 \\
NT-ProBNP (pg/mL) & 94 \\
IgG (mg/dL) & 2060.00 \\
IgA (mg/dL) & 330.0 \\
IgM (mg/dL) & 22.2 \\
\hline Light chain (kappa) (mg/dL) & 56.94 \\
Light chain (lambda) (mg/dL) & 36.65 \\
\hline Kappa/Lambda ratio & 1.55 \\
Serum and urine electrophoresis & Absence of M-protein \\
\hline
\end{tabular}

Hb, hemoglobin; WBC, white blood cells; BUN, blood urea nitrogen; NT-ProBNP, N-terminal pro-B-type natriuretic peptide; Ig, immunoglobulin.

A biopsy of enlarged inguinal lymph nodes confirmed amyloidosis (Fig. 2).

A hematological specialist conducted biopsies of bone marrow and subcutaneous fat from the abdomen to exclude multiple myeloma, and evidence of multiple myeloma and amyloidosis was not found. However, plasma cells were stained with CD138 positive, lambda positive, and CD19 negative, and were distinct from normal plasma cells. Biopsy of the lymph node showed positive staining for Congo red, indicating amyloid deposition. However, amyloid A protein was not revealed in stains of the lymph node. Patient did not have any disease to cause secondary amyloidosis, such as rheumatoid arthritis, inflammatory bowel disease, or familial Mediterranean fever.

The patient was admitted for management of lymphedema. Both legs were dressed daily with betadine soap and metronidazole powder application, and antibiotics were applied intravenously for 10 days. With consultation from hematology, chemotherapy (oral thalidomide + intravenous dexamethasone therapy) for primary amyloidosis management was begun. Redness and dis- 

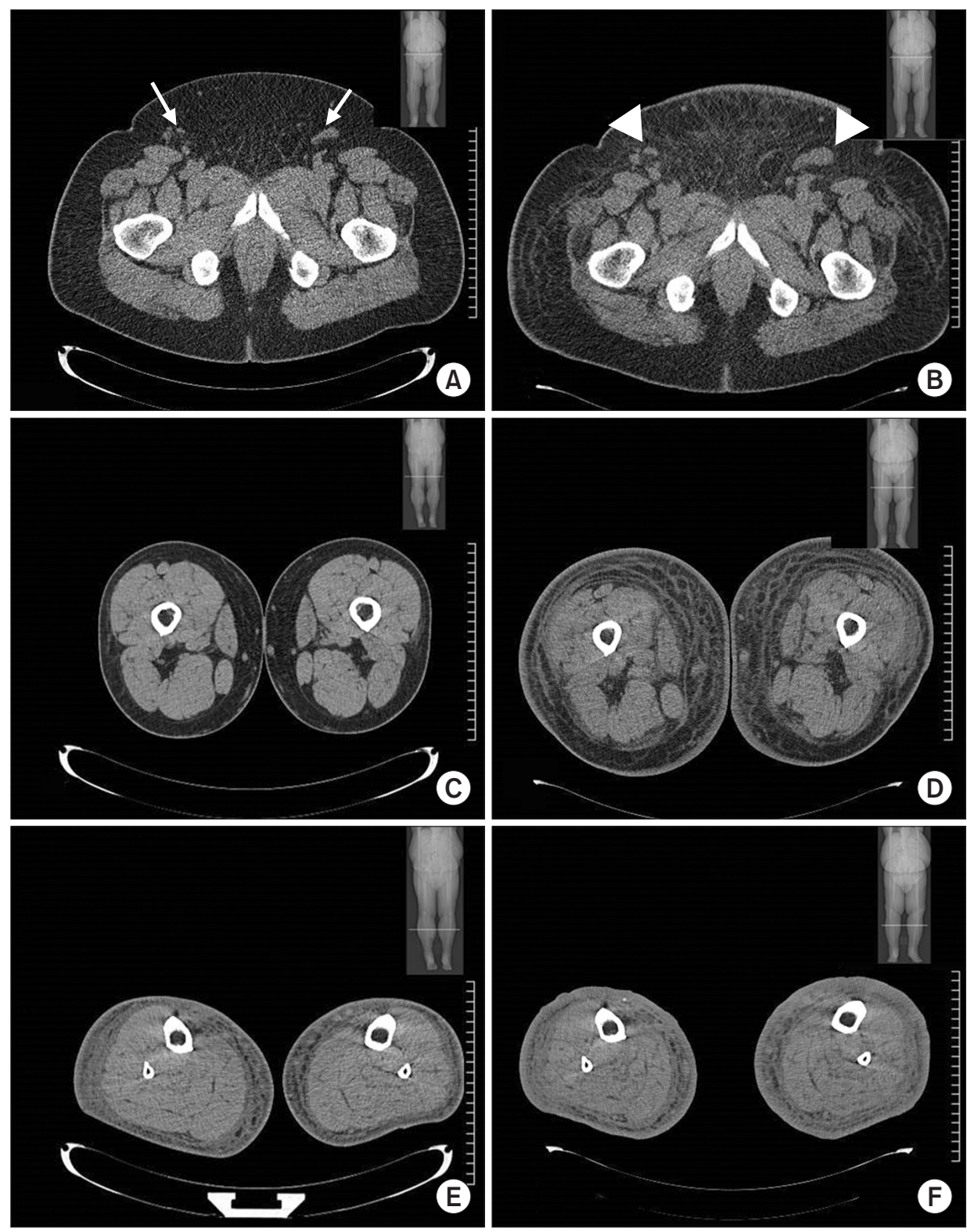

Fig. 1. Compared with previous lower extremities computed tomography (CT) scan taken 3 years ago (A, arrows), lymph nodes along the para-aortic and bilateral inguinal areas were enlarged at admission (B, arrowheads). The lower extremities CT showed subcutaneous edema in both lower extremities (C, E), and it was aggravated in follow-up CT (D, F). (C, D) above knee $10 \mathrm{~cm},(\mathrm{E}, \mathrm{F})$ below knee $10 \mathrm{~cm}$.

charges with foul odor were improved (Fig. 3). After 4 weeks of treatment, swelling in both lower extremities was improved and thigh circumference was decreased by $15 \mathrm{~cm}$ on left side and $12 \mathrm{~cm}$ on right side (measured 10 $\mathrm{cm}$ above knee). The measurements $10 \mathrm{~cm}$ below knee decreased $7 \mathrm{~cm}$ and $8 \mathrm{~cm}$ on left and right, respectively. Lymphoscintigraphy obtained 1 hour after injection of radionuclide showed no lymphatic flow in the left lower extremity and decreased lymphatic drainage in right low- er extremity, confirming the dysfunction of the lymphatic transport system in both lower extremities (Fig. 4).

\section{DISCUSSION}

We reported a patient having lymphedema in his lower extremities accompanied with primary amyloidosis involving multiple lymph nodes in aortocaval, para-aortic, common iliac, external iliac, inguinal, and femoral areas. 

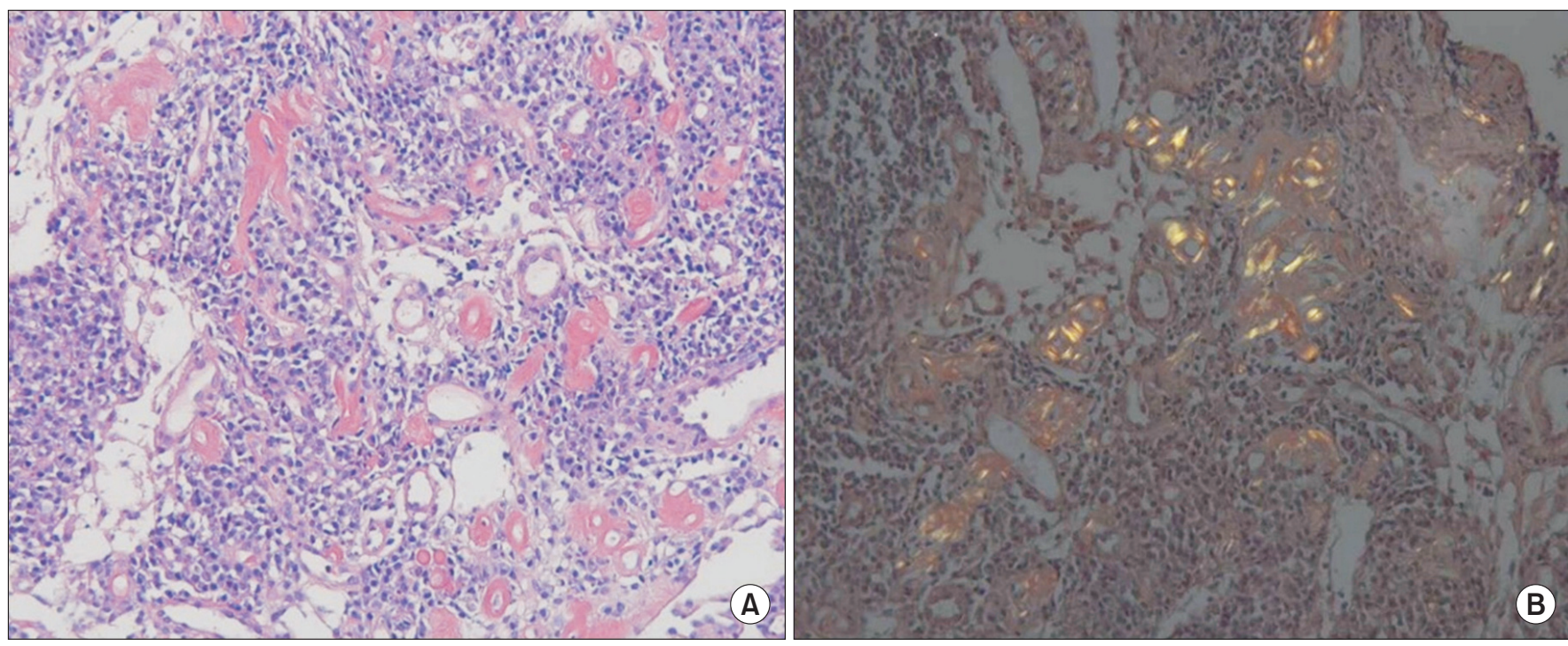

Fig. 2. Amyloid deposits were found in hematoxylin \& eosin (H\&E) and Congo red staining of specimens from lymph nodes at diagnosis (histological section). Perivascular amorphous hyaline deposition and thickening of vessel walls on H\&E staining (A) and apple-green birefringence on Congo red histological staining (B) were observed.
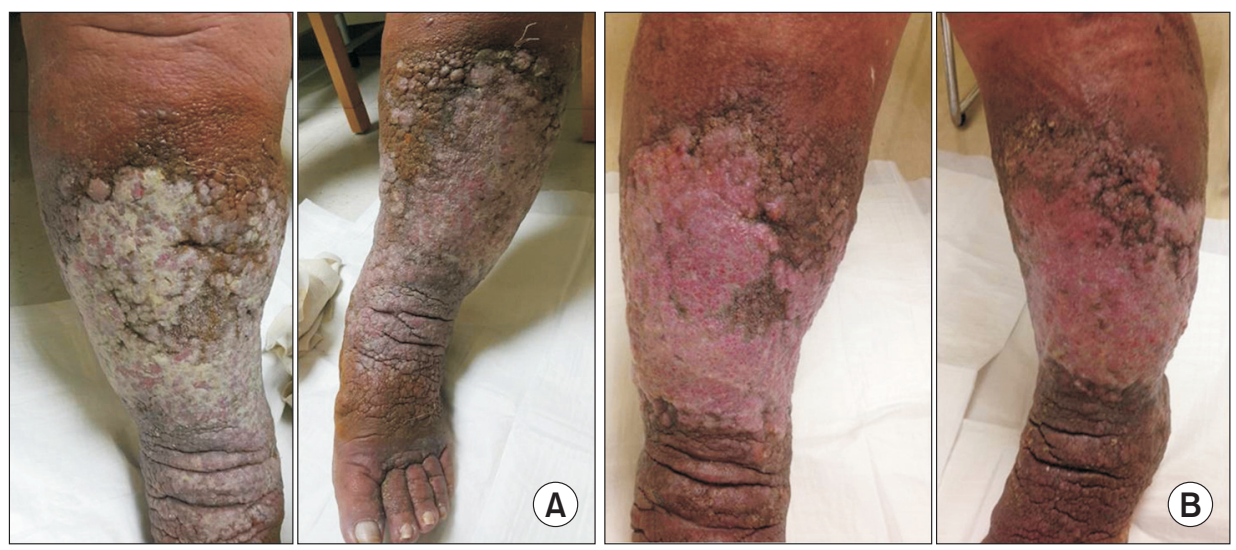

Fig. 3. Severe swelling, redness, and discharges with foul odor were exhibited at admission (A). After 4 weeks of treatment, symptoms had improved (B).

To our knowledge, this is the first report of lymphedema presenting with primary amyloidosis in Asia, though there were a few reported cases of lymphedema accompanied with primary amyloidosis in Europe [4], such as Halm et al. [5] which reported a patient with lymphedema and the deposition of amyloidosis in the mesentery and retro-peritoneum. They proposed that amyloidosis caused lymphedema by compressing lymph vessels; however, they were not able to find lymph node enlargement in the abdomen or pelvis CT scans. In our study, we found multiple enlarged lymph nodes along the paraaortic and bilateral inguinal area, and we confirmed the deposition of amyloid in the lymph nodes. It suggests that obstructing the lymph vessels by node enlargement could lead to lymphedema in bilateral lower extremities. The initial lower extremities CT scan did not reveal enlarged lymph nodes. Subsequently, swelling progressed, even to the scrotum, and legs were thickened. In followup CT scans of the lower extremities, there was para-aortic and inguinal lymph node enlargement. At this time, abdomen and pelvis CT scans showed enlarged lymph nodes not only in the para-aortic and inguinal area, but also in the aortocaval area, which was not scanned by the lower extremities CT.

In this case, amyloidosis was confirmed by finding positivity in Congo red staining in specimens of lymph node. Immunohistochemistry revealed the amyloidosis stained equivalent for antibodies of the immunoglobulin 


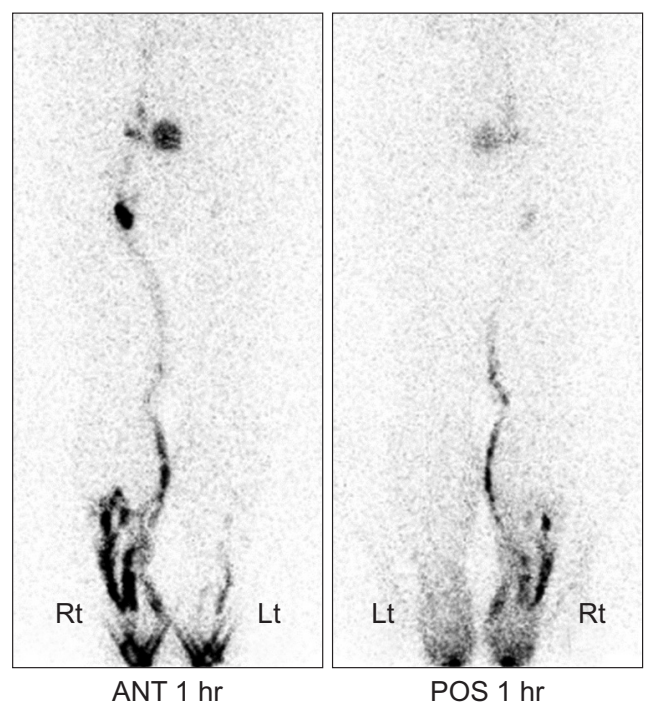

Fig. 4. In lymphoscintigraphy obtained 1 hour after injection of radionuclide there was no visualization of main lymphatics in left lower extremity. Decreased lymphatic drainage and clearance of tracer was seen in right lower extremity. Rt, right; Lt, left; ANT, anterior view; POS, posterior view; 1HR, 1 hour after injection of radionuclide.

light chain in bone marrow, but negative in lymph node. Meanwhile, the amyloidosis stained negative for antibodies of amyloid A protein in lymph node. Patient did not have any disease to cause secondary amyloidosis; thus this case was considered primary amyloidosis. The Mprotein was not observed in serum or urine. A substantial proportion of primary amyloidosis patients have no obvious M-protein, and it is possible in $10 \%$ of amyloidosis patients [6].

The lymphedema in this case could not be conclusively determined as primary or secondary. It could be that primary lymphedema entailed with AL amyloidosis, but this could also be a result of secondary lymphedema. However, primary lymphedema is very rare, with only
1.2 cases per 100,000 population [3]. This case suggested the possibility that amyloidosis lead to disruption of the circulation of lymph. In a lymphedema patient with no underlying cause, amyloidosis should be considered as one possible differential diagnosis. Primary amyloidosis is usually treated with chemotherapy, suggesting that if primary amyloidosis leads to lymphedema, complex decongestive therapy as well as chemotherapy could be essential for management of lymphedema.

\section{CONFLICT OF INTEREST}

No potential conflict of interest relevant to this article was reported.

\section{REFERENCES}

1. Wechalekar AD, Gillmore JD, Hawkins PN. Systemic amyloidosis. Lancet 2016;387:2641-54.

2. Kyle RA, Linos A, Beard CM, Linke RP, Gertz MA, O'Fallon WM, et al. Incidence and natural history of primary systemic amyloidosis in Olmsted County, Minnesota, 1950 through 1989. Blood 1992;79:1817-22.

3. Maclellan RA, Greene AK. Lymphedema. Semin Pediatr Surg 2014;23:191-7.

4. Murdaca G, Cagnati P, Gulli R, Spano F, Puppo F, Campisi $\mathrm{C}$, et al. Current views on diagnostic approach and treatment of lymphedema. Am J Med 2012;125:134-40.

5. Halm U, Berr F, Tannapfel A, Kloppel R, Secknus R, Mossner J. Primary amyloidosis of the mesentery and the retroperitoneum presenting with lymphedema. Am J Gastroenterol 1998;93:2299-300.

6. Muller AM, Geibel A, Neumann HP, Kuhnemund A, Schmitt-Graff A, Bohm J, et al. Primary (AL) amyloidosis in plasma cell disorders. Oncologist 2006;11:824-30. 